\title{
Réponse à Wander Lowie : L'émergentisme, la recherche sur l'acquisition des langues et la didactique des langues étrangères
}

\section{Georges Daniel Véronique}

\section{(2) OpenEdition}

Édition électronique

URL : http://journals.openedition.org/rdlc/1385

DOI : $10.4000 /$ rdlc. 1385

ISSN : 1958-5772

Éditeur

ACEDLE

\section{Référence électronique}

Georges Daniel Véronique, «Réponse à Wander Lowie : L'émergentisme, la recherche sur l'acquisition des langues et la didactique des langues étrangères », Recherches en didactique des langues et des cultures [En ligne], 14-1 | 2017, mis en ligne le 14 janvier 2017, consulté le 19 avril 2019. URL : http:// journals.openedition.org/rdlc/1385; DOI : 10.4000/rdlc.1385

Ce document a été généré automatiquement le 19 avril 2019

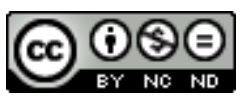

Recherches en didactique des langues et des cultures is licensed under a Creative Commons AttributionNonCommercial-NoDerivatives 4.0 International License 


\title{
Réponse à Wander Lowie : L'émergentisme, la recherche sur l'acquisition des langues et la didactique des langues étrangères
}

\author{
Georges Daniel Veronique
}

\section{Introduction}

1 L'émergentisme est une proposition philosophique relativement ancienne - certains auteurs la font remonter à John Stuart Mill (1806-1873) (Bourgine \& Engel, 1998) - mais son utilisation dans les recherches sur l'acquisition des langues étrangères et en didactique des langues est récente. Dans les travaux d'expression anglaise consacrés à la "Second Language Acquisition " ou à l'« Applied Linguistics ", il n'est guère fait mention de l'émergentisme avant les années 2000. Cependant, des notions et disciplines qui lui sont associées, sont présentes dans différentes sphères de la recherche en sciences humaines depuis une période un peu plus ancienne. En psychologie, sans remonter jusqu'au constructivisme piagétien (cité par Lowie, dans sa contribution ici même), un débat entre une vision symbolique de la cognition et la perspective sub-symbolique du connexionnisme - polémique parallèle à celle entre les théories innéistes et les théories émergentistes de l'acquisition - est en cours depuis les années quatre-vingt-dix (Fodor \& Pylyshyn, 1988 ; Smolensky, 1991). De même, les références à la théorie du Chaos et aux systèmes adaptatifs complexes, voire aux grammaires de construction, sont attestées depuis une bonne dizaine d'années en sciences du langage et ailleurs. Dans la même période, dans le secteur des travaux sur l'acquisition des langues premières, se développent des démarches fondées sur l'«usage» et sur diverses formes de constructivisme (Tomasello, 2003).

2 L'existence récente de courants émergentistes dans les recherches sur l'acquisition des langues étrangères ${ }^{1}$ invite à s'interroger sur les conditions de leur apparition, à les situer 
parmi les cadres théoriques concurrents, et enfin, à réfléchir à la signification épistémologique de cette théorisation. Dans cette réponse à l'article de W. Lowie (ici même), je voudrais reprendre et prolonger son interrogation : est-ce que l'émergentisme est une métathéorie ou une nouvelle théorie de l'acquisition linguistique? Tout d'abord, je souhaite situer succinctement le projet des approches émergentistes, en m'attachant particulièrement à la théorie des systèmes dynamiques complexes (Complex Dynamic Systems Theory, désormais CDST), au sein des théories de l'acquisition des langues étrangères, en complément de la présentation qu'en donne W. Lowie. Ensuite, je voudrais aborder la question du recours aux théories émergentistes au-delà des travaux acquisitionnistes, en didactique des langues.

\section{Les théories émergentistes au sein des recherches sur l'acquisition des langues étrangères}

3 L'apparition d'un champ de recherche consacré à l'étude de l'acquisition des langues étrangères (LE) a été fortement marquée par la proposition chomskyenne qu'une théorie du langage humain constitue également une théorie de son acquisition. Cette vision des langues humaines et de leur transmission a conduit la discipline naissante des recherches sur l'acquisition des langues étrangères (RAL) à se nourrir des travaux sur l'acquisition des langues premières (désormais L1) et, dans un premier temps du moins, d'un chomkysme diffus. Les recherches sur l'acquisition des langues premières se sont assez vite affranchies de l'innéisme chomskyen pour se diriger vers d'autres démarches (Halliday, 1975 ; Karmiloff-Smith, 1979 ; Slobin, 1985 ; Bates \& McWhinney, 1987 ; etc.). Il en a été de même pour l'étude de l'acquisition des langues étrangères. C'est dans ce contexte qu'il convient d'examiner les propositions émergentistes, dont celle de la CDST. Cette section devrait permettre de mieux cerner la nature et la portée de cette entreprise théorique.

\section{Théories et modèles de l'acquisition des langues étrangères}

Toute théorie de l'acquisition des langues étrangères se doit de répondre à tout ou partie des questions suivantes :

- quelle est la nature du point de départ (initial state) de l'apprentissage d'une langue étrangère? Comment caractériser l'usage de la faculté de langage et des langues antérieurement connues, dont la L1, dans l'appropriation d'une LE ?

- comment décrire le développement des connaissances en LE et ses déterminants ? Existe-il un point terminal (ultimate state) de l'apprentissage d'une LE ?

- quel rôle réserver aux interactions verbales et sociales dans l'analyse de l'appropriation d'Une LE?

Chacun des quelques quarante théories et modèles que recensent Larsen-Freeman \& Long (1991: 227) dans le champ des recherches sur l'acquisition des langues étrangères éclairent certains aspects des questions posées supra en fonction de leurs postulats et de leurs choix épistémologiques.

6 Long (2000) montre que les théories de l'acquisition des LE diffèrent selon les cinq critères suivants : i) leur source - sont-elles élaborées à partir de données empiriques (voir par exemple: Klein \& Perdue, 1997 et leur hypothèse de la variété de base) ou 
empruntées à un autre champ (voir les théories ayant recours à la grammaire universelle (GU), ou la référence à la théorie mathématique du Chaos dans la CDST) ?, ii) leur amplitude - quelle est la nature des phénomènes expliqués ? (Dans la cas de la CDST, tous les aspects linguistiques systémiques sont abordés sous l'angle de la variabilité individuelle), iii) leur contenu - quels sont les mécanismes explicatifs invoqués ?, iv) leur type - Long distingue deux familles de théories, le camp nativiste et le camp empiriciste et v) la nature de leurs propositions - sous forme de lois ou sous forme de relations entre cause et processus comme dans la théorie de la processabilité de Pienemann (1998).

7 Comme l'indique la contribution de W. Lowie ici même (mais voir également infra), l'émergentisme de la Complex Dynamic Systems Theory récuse l'idée qu'il existe un état initial ou final de l'acquisition des langues étrangères. Elle analyse des observables linguistiques, recueillies selon les méthodes en cours dans les travaux en acquisition, en se focalisant sur la variabilité inter- et intra-individuelle. Tout en analysant les mêmes observables que les théories cognitivistes concurrentes (Long, 2000), cette théorie se situe sur un autre plan épistémologique en tant que proposition de modélisation et de théorisation de l'acquisition d'une langue étrangère.

\section{Confrontations théoriques dans les recherches sur l'acquisition des langues étrangères}

8 La formulation de l'hypothèse de l'interlangue (ou variété linguistique transitoire de l'apprenant en LE), dans les années 70 du vingtième siècle (Selinker, 1972), provoque la confrontation de deux ensembles de positions théoriques : l'un inspiré par la grammaire générative considère que des traits spécifiques distinguent l'interlangue des autres langues naturelles humaines, et l'autre, dérivé de la sociolinguistique variationniste de W. Labov, soutient que les caractères qui différencient apparemment l'interlangue des autres systèmes linguistiques sont de fait partagés par ces derniers (Tarone, 1988). Les débats des années 70-80, entre les démarches rationalistes cognitives et les travaux à orientation sociale et pragmatique, dans l'étude du procès d'acquisition des L2 prennent un tour plus virulent avec la parution d'un numéro du Modern Language Journal en 1997, qui conteste les approches cognitivistes de l'acquisition. Les partisans des approches socioculturelles y appellent de leurs vœux une nouvelle conceptualisation des recherches sur l'acquisition des langues étrangères (Firth \& Wagner, 1997). Ils militent en faveur d'un élargissement des thèmes de recherche qui rendent compte de la multi-dimensionnalité de la socialisation en L2, et remettent en cause des études centrées sur la cognition individuelle plutôt que sur une cognition socialement située et partagée.

$9 \mathrm{Au}$ cœur de ces débats se trouve une confrontation entre les théories socioculturelles (environnementaliste et interactionniste dans la terminologie de Larsen-Freeman \& Long, 1991) et les théories cognitives et neuronales de l'acquisition des L2, dont certaines sont nativistes. Larsen-Freeman (2007) résume les divergences entre les théories socioculturelles de l'acquisition des LE et les théories cognitivistes en douze points. Elle souligne, entre autres : i) la place différente attribuée au contexte dans les deux types de démarches ; ii) la représentation divergente du langage humain et de l'objet de recherche dans ces deux ensembles théoriques ; iii) leurs représentations différentes de l'apprenant et de son identité ; et, iv) les orientations philosophiques et épistémologiques divergentes des deux ensembles théoriques. Les théories cognitivistes, à leur tour, rejettent les théories socioculturelles en arguant qu'elles se méprennent sur l'objet d'une théorie de 
l'acquisition, et en critiquant leurs choix épistémologiques, notamment leur constructivisme, et leurs méthodologies de recherche (Gregg, 2005). Ces débats sont à l'origine d'un tournant social (social turn) dans les recherches sur l'acquisition des langues étrangères (Block, 2003). La CDST s'inscrit dans la dynamique ascendante des démarches socioculturelles, par sa conception du langage et des langues et par son rejet de l'innéisme (voir Véronique, 2013).

\section{L'émergentisme parmi les approches socioculturelles}

Depuis la fin des années 90, les théories socioculturelles de l'acquisition des langues étrangères s'affirment dans leur diversité. L'on peut ranger sous cette vaste bannière, les approches écologiques de l'acquisition de L2 (Kramsch \& Vork Steffessen, 2010), les démarches en termes de socialisation (Norton \& Toohey, 2002), la théorie socioculturelle vygotskyenne (Lantolf, 2006), la théorie critique et l'acquisition des LE (Watson-Gegeo \& Nielsen, 2005), l'analyse conversationnelle et l'acquisition des LE (Mondada \& PekarekDoehler, 1992), l'approche sociolinguistique (Tarone, 1987) et la théorie émergentiste (MacWhinney, 2006). Toutes ces démarches théoriques insistent sur le fait qu'il faut étudier l'acquisition des LE comme une activité sociale et culturelle. Il convient de la penser comme un accomplissement social, en retenant le point de vue des apprenants qui sont des acteurs sociaux. Les relations de l'apprenant et du social doivent être envisagées en termes de relations de pouvoir, d'enjeux identitaires et de communautés de pratiques.

11 Tout en s'inscrivant dans une perspective socioculturelle, les théories de l'émergentisme, dont la CDST, semblent partager en propre, les idées suivantes :

- le développement de l'acquisition des L1 et des LE est graduel et global,

- en matière d'input, les cas exemplaires (exemplars) jouent un rôle fondamental dans la mise en place des régularités en LE,

- le connexionisme propose la conceptualisation la plus adéquate du rapport cerveau (brain) cognition (mind).

Elles manifestent également une relative défiance à l'égard du positivisme et du rationalisme et adhérent à des modèles de la complexité (Larsen-Freeman \& Cameron, 2008).

\section{Une analyse de la contribution de W. Lowie}

13 W. Lowie inscrit la théorie qu'il défend au cœur des approches « Usage-based » telles que présentées par Tomasello (2003) et MacWhinney (2006). A partir de l'idée qu'une langue s'acquiert à travers l'input et l'interaction communicative, il pose que les seules capacités cognitives de l'attention métalinguistique (noticing) et de la catégorisation (categorization) suffisent à provoquer l'émergence de nouvelles connaissances en langue étrangère lors d'un apprentissage. L'apport propre de la CDST est d'insister sur la dimension individuelle de tout apprentissage linguistique et sur la variabilité des démarches individuelles. Cela conduit W. Lowie à poser que c'est le degré de variabilité élevé des productions d'apprenants qui constitue le moteur de la dynamique d'interaction et d'évolution des sous-systèmes des interlangues. Des attracteurs constitués des soussystèmes de L1 et de L2 sous-tendent la dynamique de la variété linguistique émergente employée par l'apprenant. Au terme de sa contribution, W. Lowie s'interroge sur les implications de la démarche émergentiste pour l'enseignement des langues étrangères. 
Les propositions qu'il formule ne sont pas sans intérêt mais paraissent assez limitées en didactique des langues étrangères par leur seule insistance sur le lien entre emploi et apprentissage de la LE et sur la dimension individuelle de l'apprentissage.

Depuis, les travaux de Tarone (1988), la variabilité des activités des apprenants en LE est au cœur de nombreuses propositions théoriques en RAL. Indéniablement la CDST que présente $\mathrm{W}$. Lowie fournit une modélisation mathématique adéquate des comportements individuels et variables des apprenants de LE. L'objection majeure que l'on peut faire à l'encontre de cette démarche tient au fait qu'en dépit de la variation inter-individuelle, de nombreux travaux en RAL ont dégagé des stades dans l'appropriation des LE (voir par exemple, Pienemann, 1998 ou Bartning \& Schlyter, 2004). Que faire des stades d'acquisition dégagés pour une langue cible déterminée et des séquences de développement que les recherches sur l'acquisition de LE ont mis à jour? Est-ce que ce sont seulement des artefacts, sans liens avec le réel des acquisitions?

L'apport propre de la CDST est de contester les représentations de la dynamique d'acquisition linguistique qui lissent les variations et les régressions des interlangues, au bénéfice de représentations qui modélisent le changement de façon plus précise. D'ailleurs, l'ouvrage collectif de Verspoor, de Bot \& Lowie (2011: 129-199) consacrent environ une centaine de pages aux techniques de traitement, d'analyse des données en LE et à leur modélisation statistique.

\section{Matrice disciplinaire et méta-théorie}

'on compare la CDST aux autres modèles émergentistes et socioculturels, et au-delà, aux autres théories et modèles ayant cours dans le champ de la recherche sur l'acquisition des langues secondes, ce qui caractérise cette proposition, c'est qu'elle apparait tardivement. En un sens, la CDST est sans doute la dernière des grandes théories à être formulée dans le domaine de la RAL. Cela étant posé, la CDST s'insère dans une «matrice disciplinaire » particulière, qu'elle partage d'abord avec les théories et modèles socioculturels (Dumouchet, 2002). Elle ne s'en distingue que par la forme que prend la représentation des données qu'elle analyse et par la mise en forme de ses résultats, où le recours à la statistique et à la modélisation mathématique la sépare des approches plus qualitatives.

17 Du fait de ce hasard chronologique, il serait tentant de considérer la CDST comme une méta-théorie. Cependant elle ne satisfait pas aux critères qu'énonce Aguirre Oraá (2002 : 1625) dans sa définition de la métathéorie :

18 Le terme désigne une théorie qui analyse les structures et les méthodes d'autres théories, une théorie qui a pour objet les caractéristiques structurelles des théories qui interviennent en science, quelle qu'en soit la nature particulière: une théorie sur les théories. [...] la métathéorie étudie le système des postulats et des concepts des différentes théories, établit les limites d'application des théories, et analyse leurs moyens d'expression linguistique, en considérant leur contenu empirique ou en s'assurant de leur cohérence logique.

En effet, même si la CDST s'inscrit dans une démarche polémique à l'égard de certaines théories cognitivistes, elle revendique, au contraire, sa filiation avec les autres théories émergentistes avec lesquelles elle partage des perspectives identiques sur les langues comme systèmes adaptatifs complexes (Verspoor, de Bot \& Lowie (eds), 2011 : 29-38). 


\section{Émergentisme et didactique des langues étrangères}

Dans la compétition entre modèles et théories de l'acquisition des langues étrangères, l'émergentisme, dans la version de la CDST, renouvelle la description des variétés d'apprenants, par sa modélisation de la variabilité des données d'apprenants. Quelles sont les implications de cette théorie, au-delà des recherches en acquisition, dans le champ plus vaste de la Didactique des langues étrangères (DLE) ? La DLE, comme tout domaine de recherche, est traversée par diverses propositions théoriques et méthodologiques qui informent ses analyses, fournissent des grilles de lecture et d'interprétation de ses observations et de ses pratiques d'intervention et leur donnent sens. Le dialogue entre la DLE et les recherches sur l'acquisition des langues étrangères, qu'elles soient d'orientation socioculturelle ou cognitive (voir supra) est difficile. Cela tient aux différences d'orientation et de finalité de ces disciplines, à des thématiques de recherche divergentes, à des effets de doxa indissociables des activités auxquelles se livrent didacticiens et linguistes et à des différences de mode de démonstration et d'argumentation. Le point de contentieux entre ces disciplines réside assurément dans la nature de la référence à la linguistique et de l'usage fait de cette discipline en DLE et en RAL (Véronique, 2005).

\section{La DLE et la RAL}

En tant que discipline consacrée aux situations éducatives d'enseignement et d'apprentissage, la didactique des langues étrangères se préoccupe certainement de questions, au moins partiellement, différentes de celles de la RAL. La discipline technologique de la didactique fédère des activités variées : des questions curriculaires, des choix méthodologiques et pédagogiques et des interrogations autour des cultures éducatives, linguistiques et des pratiques didactiques. Au contraire de la discipline composite allemande Sprachlehr und Lernforschung (recherche sur l'enseignement et l'apprentissage des langues) (Edmondson \& House, 2000) - qui semble être dorénavant en mauvaise posture - ou de la second language classroom research (recherche sur les classes de langues étrangères) (cf. Nunan, 1991), aucune discipline intermédiaire n'existe dans le domaine francophone entre les recherches en acquisition et en didactique des langues étrangères. En dépit d'une proposition de Bouchard (1992), un secteur de recherche comparable à ceux qui se sont affirmés en contexte germanophone ou anglophone, unissant les travaux des didacticiens et des acquisitionnistes en un nouvel ensemble, distinct de la RAL, n'a pas émergé, en France du moins. Cela s'explique partiellement par l'absence de dialogue institutionnel, voire scientifique, entre la recherche universitaire et les instances de formation de formateurs.

On peut penser que les travaux orientés vers l'étude de la socialisation en langue étrangère offrent davantage d'ouvertures vers les préoccupations de recherche en DLE. Les approches émergentistes en acquisition des LE pourrait constituer des passerelles qui permettraient à certains secteurs de la DLE de nouer un dialogue avec la RAL. 


\section{L'émergentisme en DLE}

23 On peut envisager deux rôles non exclusifs pour l'émergentisme en didactique : l'un serait de permettre la jonction entre la didactique et la recherche en acquisition, et l'autre de fournir la matrice disciplinaire d'une nouvelle approche en DLE. Eu égard à ce qui a été exposé supra à propos des confrontations entre modèles et théories dans le champ des recherches sur l'acquisition des langues étrangères, les approches socioculturelles de l'acquisition des LE semblent davantage accessibles aux interrogations de la didactique des LE. Cela tient précisément au tournant social qui a conduit ces théories de la socialisation en LE à se détourner des approches formalistes en sciences du langage, et à privilégier l'ethnographie de la communication, l'analyse conversationnelle ou encore discursive dans leur conceptualisation des activités langagières en LE.

Si des démarches émergentistes en acquisition constituent de bons candidats pour des échanges avec la DLE, l'on doit ajouter cependant que de l'ensemble de ces propositions, la CDST est celle qui semble la moins immédiatement accessible. En effet, cette version de l'émergentisme reste proche par bien des aspects - certaines valeurs et certaines formulations - de la matrice disciplinaire (Dumouchel, 2002) des théories cognitivistes en RAL.

Que peut apporter l'émergentisme, et tout particulièrement la CDST, en DLE ? Peut-il caractériser utilement la somme des phénomènes engagés dans les procès d'enseignement et d'apprentissage des langues? Est-il susceptible de mettre les perspectives cognitives, numériques et méthodologiques qui alimentent ses travaux en acquisition, au service d'une perspective plus holistique en didactique?

Comme d'autres approches en acquisition des langues étrangères, l'émergentisme en didactique des langues étrangère peut aider à expliquer les progrès des apprenants, voire à mieux comprendre la cognition enseignante (teacher cognition). Il est moins certain que cette approche, dans sa version CDST notamment, puisse contribuer aux activités curriculaires en DLE, à la structuration de la matière à enseigner, à la définition des tâches d'enseignement ou à la mise en place de conduites pédagogiques en classe de langues.

On pourrait se demander si l'apport propre de l'émergentisme en didactique des langues étrangères n'est pas de remettre en question certaines pratiques traditionnelles en DLE en y faisant advenir les travaux acquisitionnistes. Pour ma part, je ne considère pas que la DLE doive être contestée en tant que discipline mais j'admets volontiers qu'elle peut être traversée par des polémiques, voire des pratiques divergentes. Ainsi, on peut considérer que la pratique institutionnelle de la DLE est autoritaire.

D'ailleurs, c'est le sens de s'interroger sur la pratique d'une didactique émergentiste.

Peut-on être émergentiste en didactique comme on est, par exemple, constructiviste ?

Dans son épilogue à A dynamic Approach to Second Language Development. Methods and Technique, K. de Bot signale deux usages du terme "émergentisme ", l'un métaphorique, usage qu'il attribue à Kramsch et Van Lier, et, l'autre plus précis, qui rapproche la CDST des modèles mathématiques dont elle s'inspire. Je me rallierai volontiers au point de vue qu'exprime implicitement de Bot pour dire que le terme 'didactique émergentiste', n'a de sens que d'une façon assez superficielle. Ce label pourrait indiquer alors qu'il s'agit d'une pratique de la DLE qui partage certaines des valeurs de l'émergentisme: son antiinnéisme et son adhésion à la pensée «complexe». On pourrait soutenir que le terme 
'didactique émergentiste' dont je ne me revendique nullement, contient une " contradiction dans les termes ». Pour ma part, je ne suis pas sûr que ce que recouvre la discipline de la didactique soit incompatible avec la matrice disciplinaire que représente l'émergentisme. Je suis sensible au fait que ce dernier paradigme fait sens dans un débat en RAL comme j'ai tenté de le montrer supra et moins en DLE.

\section{Conclusion}

La CDST, en tant que théorie et modèle émergentistes qui souhaitent rendre compte de l'évolution dynamique des interlangues d'apprenants dans leur variabilité, suscite des réserves de ses modèles concurrents en RAL. Au-delà des débats dans le domaine des études de l'acquisition des langues étrangères, l'émergentisme peut-il offrir une matrice disciplinaire à la DLE ? On conçoit volontiers que des valeurs et des thèses que partagent les émergentismes, et au-delà les approches socioculturelles en RAL, puissent intéresser la DLE (Larsen-Freeman \& Cameron, 2008). En effet, la nature praxéologique de cette dernière discipline devrait la rendre sensible à la complexité de la socialisation en langue étrangère.

Cependant, sous bénéfice d'inventaire, tout indique que l'avènement d'une «didactique émergentiste ", relève davantage de la métaphore séduisante, voire séductrice, plutôt que de l'avancée scientifique.

31 Si contradiction il y a dans "didactique émergentiste " - appellation que je conteste -, elle tient à la volonté de rapprocher indûment une modélisation de l'acquisition linguistique, et une discipline qui aborde une myriade de questions liées, entre autres, aux institutions éducatives qui s'occupent d'enseignement des langues. Des proximités conceptuelles, philosophiques, voire épistémologiques dans la recherche sur l'acquisition linguistique et dans les recherches en DLE ne sauraient justifier à elles seules, la mise en avant d'une « didactique émergentiste ». Cela me semble particulièrement flagrant si l'on se tourne vers la théorie et la pratique de la Complex Dynamic Systems Theory. En effet, cette variété de démarche émergentiste, qui n'est pas sans contradiction dans ses efforts de modélisation (De Bot 2011 : 126), pratique une démarche quantitative, tout en partageant des valeurs qui sont celles de démarches qualitatives. Si cela se défend en RAL, la transposition de l'émergentisme, notamment de la CDST, en DLE me semble plus aléatoire.

\section{BIBLIOGRAPHIE}

Aguirre Oraá, J.M. (2002). « Métathéorie ». In Auroux, S. (dir.). (2002). Les notions philosophiques. Dictionnaire. Paris : P.U.F. 3e édition, pp. 1625.

Bartning, I. \& Schlyter, S. (2004). «Itinéraires acquisitionnels et stades de développement en français L2 ». Journal of French language Studies. vol. 14, 1, pp. 281-299. 
Bates, E. \& MacWhinney, B. (1987). S« econd language acquisition from a functionalist perspective : Pragmatic, semantic and perceptual strategies ». In Winitz, H. (ed). « Native Language and Foreign Language Acquisition ». Annals of the new York Academy of Sciences. Vol 379, pp. 190-214.

Block, D. (2003). The Social Turn in Second Language Acquisition. Edinburgh : Edinburgh University Press.

Bouchard, R. (1992). « Présentation », dans Bouchard, R.et al. (éd.), Acquisition et enseignement/ apprentissage des langues. Grenoble, Lidilem. pp. 5-13

Bourgine, P. \& Engel, P. (1998). « Emergence ». In Houdé, O. et al. Vocabulaire de sciences cognitives. Paris : PUF. pp. 152-154.

Dumouchel, P. (2002). « Paradigme ». In Auroux, S. (dir.). 2002. Les notions philosophiques. Dictionnaire. Paris : P.U.F. $3^{\text {ème }}$ édition. pp. 1847-48.

De Bot, K. (2011). « Epilogue ». In Verspoor, M.H, de Bot, K., Lowie, W. (eds.) 2001. A dynamic Approach to Second Language Development. Methods and Techniques.. Amsterdam : J. Benjamins. pp. 123-127.

Edmondson, W. \& House, J. (2000). « Einführung in die Sprachlehrforschung ». 2. Auflage.Tübingen \& Basel : A. Francke.

Firth, A. \& Wagner, J. (1997). O« n Discourse, Communication and (some) Fundamental Concepts in SLA Research ». Modern Language Journal. 81, pp. 285-300.

Fodor, J.A. \& Pylsyshyn, Z.W. (1988). « Connectionism and cognitive architecture : a critical analysis ». Cognition, 28, pp. 3-71.

Gregg, K. (2005). « SLA : Theory Construction and Assessment ». In Doughty, C.J. \& Long, M. (eds.). The Handbook of Second Language Acquisition. Oxford : Blackwell. pp. 831-865

Halliday, M.A.K. (1975). « Learning how to mean : Explorations in the development of language ». London: Edward Arnold.

Karmiloff-Smith, A. (1979). A functional approach to child language. Cambridge : C.U.P.

Klein, W. \& Perdue, C. (1997). « The Basic Variety (or Couldn't natural languages be much simpler?) ». Second Language Research. 13, pp. 301-347.

Kramsch, C. \& Vork Steffensen, J. (2010). « Ecological Perspectives on Second Language Acquisition ». In Duff, P.A. \& Hornberger, N.C. (eds.). Language Socialization. vol. 8. Encyclopaedia of Language and Education. New-York : Springer. pp. 17-28.

Lantolf, J. \& Thorne, S.L. (2006). « Sociocultural Theory and the Genesis of Second Language Development ». Oxford : OUP.

Larsen-Freeman, D. (2007). « Reflecting on the Cogntive-Social Debate ». Modern Language Journal. 91, pp. 773-87.

Larsen-Freeman, D. \& Long, M. (1991). An introduction to second language acquisition research. London : Longman.

Larsen-Freeman, D. \& Cameron, L. (2008). Complex Systems and Applied Linguistics. Oxford : OUP. Long, M. (2000). « Second Language Acquisition Theories ». In Byram, M. (ed.), Encyclopedia of Language Teaching and Language Learning. London : Routledge.

MacWhinney, B. (2006). « Emergentism : Use often and with care ». Applied Linguistics. vol. 27, n

${ }^{\circ} 4$, pp. 729-740. 
Matthey, M. \& Véronique, D. (2004). « Trois approches de l'acquisition des langues étrangères : enjeux et perspectives ». AILE 21, pp. 203-223.

Mondada, L. \& Pekarek-Doehler, S. (2000). «Interaction sociale et cognition située : quels modèles pour la recherche sur l'acquisition des langues? », AILE 12, pp. 147-174.

Norton, B. \& Toohey, K. (2002). « Identity and language learning ». In Kaplan, R. (ed.). The Oxford Handbook of Applied Linguistics. Oxford : OUP. pp. 115-123.

Nunan, D. (1991). « Methods in Second Language Classroom-Oriented Research : A Critical Review ». Studies in Second Language Acquisition. vol. 13, n² 2, pp. 249-274.

Pienemann, M. (1998). Language Process and Second Language Development : Processability Theory. Amsterdam : J. Benjamins.

Selinker, L. (1972). « Interlanguage ». International Review of Applied Linguistics. 10, pp. 209-231.

Slobin, D. I. (Ed.). (1985). The crosslinguistic study of language acquisition. vol. 1. The data. Hillsdale, NJ : Lawrence Erlbaum Associates.

Slobin, D. I. (Ed.). (1985). The crosslinguistic study of language acquisition. vol. 2. Theoretical issues. Hillsdale, NJ : Lawrence Erlbaum Associates.

Smolensky, P. (1991). « Connectionism, constituency and the language of thought ». In Loewer, B. \& Rey, G. (eds.) Meaning in mind : Fodor and his critics. Oxford : Basil Blackwell.

Tomasello, M. (2003). Constructing a language : A Usage-Based theory of Language Acquisition. Harvard : Harvard University Press.

Tarone, E. (1988). Variation in interlanguage. London : Edward Arnold.

Véronique, G.-D. (2005). « Les interrelations entre la recherche sur l'acquisition du français langue étrangère et la didactique du français langue étrangère ». AILE 23, pp. 9-41.

Véronique, G.D. (2013). « Socialization ». In Herschensohn, J. \& Young-Scholten, M. (eds). The Cambridge Handbook of Second Language Acquisition. Cambridge : CUP. pp. 251-271

Verspoor, M.H, de Bot, K., Lowie, W. (eds.). (2011). A dynamic Approach to Second Language Development. Methods and Techniques. Amsterdam : J. Benjamins.

Watson-Gegeo, K.A. \& Nielsen, S. (2005). « Language Socialization in SLA ». In Doughty, C.J. and Long, M. (eds.). The Handbook of Second Language Acquisition. Oxford : Blackwell. pp. 155-177.

Zuengler, J. \& Miller, E. R. (2006). « Cognitive and Sociocultural Perspectives : Two parallel SLA World ». TESOL 40, pp. 35-58.

\section{NOTES}

1. Les recherches en acquisition désignent la langue de première socialisation à l'aide de l'acronyme L1; dans le cas des situations d'acquisition bilingue, on utilisera alors 2L1. Pour l'acquisition des langues non premières, « langue seconde » (L2) ou « langue étrangère » (LE) sont usités. En didactique des langues, on distingue souvent des contextes de langue seconde, des contextes de langue étrangère. Cette distinction pertinente pour l'enseignement sera ignorée ici. 


\section{RÉSUMÉS}

En réponse à l'article de Wander Lowie, cette contribution se propose de situer la Complex Dynamic System Theory, dont il se réclame, au sein des écoles et des théories qui organisent les recherches sur l'acquisition des langues. Cette réaction rappelle brièvement quelques-uns des débats qui opposent les travaux résolument tournés vers l'appréhension cognitive et neuronale du procès d'appropriation linguistique et ceux qui s'intéressent principalement à la socialisation en langue étrangère.

Cet article s'interroge ensuite sur la façon dont les théories émergentistes de l'acquisition linguistique sont susceptibles de s'articuler avec les thèses et les pratiques qui ont cours en didactique des langues étrangères.

The discussion of the paper of Wander Lowie presented in this contribution attempts to situate the Complex Dynamic System Theory claimed by Lowie, within the schools and theories which structure the field of Second Language Acquisition Research. The paper examines briefly some of the controversies within SLA research between cognitive and neuronal theories of language acquisition and theories focused on L2 acquisition as socialization.

The paper also examines the way in which emergentist theories may contribute to the theories and practices of Language Didactics.

\section{INDEX}

Mots-clés : émergentisme, acquisition des langues, théorie socioculturelle, complexité

Keywords : emergentism, language acquisition, sociocultural theory, complexity.

\section{AUTEUR}

\section{GEORGES DANIEL VERONIQUE}

Georges Daniel Veronique est professeur des universités à l'université d'Aix Marseille. Il appartient au laboratoire 'Langage et Parole' (UMR 7309) et travaille sur l'acquisition du français langue étrangère, en didactique du FLE, sur la créolisation et les créoles français.

Courriel : georges.veronique[a]orange.fr. 